\title{
I-Gel: A Rescue Intubation Device in Unanticipated Difficult Intubation for Emergency Laparotomy*
}

\author{
Lalit Gupta $^{1 \#}$, Kapil Chaudhary ${ }^{1}$, Poonam Bhadoria ${ }^{2}$ \\ ${ }^{1}$ Department of Anaesthesia \& Intensive Care, G. B. Pant Hospital \& Associated Maulana Azad Medical College, New Delhi, India; \\ ${ }^{2}$ Department of Anaesthesia \& Intensive Care, Maulana Azad Medical College \& Associated Lok Nayak Hospital, New Delhi, India. \\ Email: "lalit.doc@gmail.com
}

Received October $18^{\text {th }}, 2011$; revised October $30^{\text {th }}, 2011$; accepted November $9^{\text {th }}, 2011$

\begin{abstract}
The "i-gel" is a newer, non-inflatable supraglottic airway device for clinical use and also for resuscitation purposes. It has also been found to be a useful ventilation and intubation device in anticipated/simulated difficult airway situations. However, its use in unanticipated difficult airway situations in emergency set up has not been explored. We describe a case of 24-year-old male posted for emergency laparotomy that turned out to have an unanticipated difficult airway. Attempts to intubation and ProSeal Laryngeal Mask Airway insertion failed. However, "i-gel" proved to be a very handy intubating conduit in this critical situation.
\end{abstract}

Keywords: I-Gel; Rescue; Unanticipated Difficult Intubation; Emergency; Laparotomy

\section{Introduction}

The i-gel (Intersurgical, Wokingham, UK) is one of the recent and useful developments in supraglottic airway devices. Whilst there has been an explosion in the development of supraglottic airway devices in recent years, i-gel is a new device with some distinctive features. This non-inflatable supraglottic device has been in use for clinical purposes and has recently been found to be additionally useful for resuscitation purposes [1]. The various trials for its use in difficult airway have reported it to be a useful ventilation and intubation device [2-4]. However, a literature search reveals its role in unanticipated difficult airway in an emergency scenario to be largely unrecognized. We describe a case of 24-year-old male posted for emergency laparotomy that turned out to have an unanticipated difficult airway where varied attempts to intubate the trachea failed and "i-gel" proved to be a rescue intubation conduit.

\section{Case History}

A 24-year-old male, weighing $60 \mathrm{~kg}$, presented in surgical emergency with the diagnosis of perforation peritonitis and was posted for emergency laparotomy. The air-

\footnotetext{
"Key Messages: This case report highlights the use of i-gel as a rescue intubation device in a patient with unanticipated difficult intubation in emergency setup in who attempts to intubation and Proseal laryngeal mask airway failed.

${ }^{\#}$ Corresponding author.
}

way assessment was normal with Mallampatti classification II.

The patient was given injection ranitidine $50 \mathrm{mg}$ i.v., 15 min prior to his transportation to the emergency operation room (EOR). Preoperative vitals of the patient were stable. After preoxygenation, patient was given thiopentone $250 \mathrm{mg}$ i.v. and succinylcholine $100 \mathrm{mg}$ i.v. and cricoid pressure was applied. The direct laryngoscopy of the patient, after adequate muscle relaxation, revealed a Cormack and Lehane (CL) grade III view, not improving even on release of cricoid pressure. Repeat laryngoscopy after repositioning of the head and using McCoy laryngoscope also revealed CL grade III b view with a relatively large epiglottis and tracheal intubation could not be achieved. ProSeal LMA size 4 could not achieve proper placement on two successive attempts. A single attempt with ProSeal LMA size 3 was also unsuccessful. Face mask ventilation was still adequate and the vitals were stable with $\mathrm{SpO}_{2}$ of $98 \%$.

The intubating LMA and fiberscope were unavailable in the EOR. So, an attempt was next made to insert i-gel size 4 after repeating succinylcholine with atropine along with $100 \% \mathrm{O}_{2}$. I-gel was appropriately placed in single attempt as adjudged by adequate chest rise and end-tidal $\mathrm{CO}_{2}$ waveform. The trachea was successfully intubated with a $7 \mathrm{~mm}$ ID cuffed tracheal tube through the stem of i-gel airway device in first attempt and tracheal cuff inflated. Cricoid pressure was released after confirmation of correct tube placement. The bevelled patient end of 
6mm ID tracheal tube was fixed in the machine end of the correctly placed $7 \mathrm{~mm}$ ID tube, after removing the universal connector, and i-gel was gently removed over it ("tube within the tube" assembly) [5]. The bilaterally equal air entry was confirmed. A $16 \mathrm{~F}$ nasogastric tube was inserted and aspiration of enteric contents was done. The anesthesia was maintained with isoflurane in, nitrousoxide (66\%), and oxygen (33\%) mixture. Vecuronium aliquots were used for maintenance of muscle relaxation. Surgery proceeded uneventfully and the patient's vitals remained stable throughout.

On completion of surgery and antagonism of neuromuscular blockade with neostigmine and glycopyrrolate, the patient was breathing spontaneously with good tidal volume. The patient was extubated over a tube exchanger when he was fully awake and responding to commands, keeping in mind the possibility of difficult extubation. The patient was observed in EOR for 30 minutes and then shifted to the post-operative recovery room and had an uneventful course.

\section{Discussion}

Quite often anaesthesiologists encounter cases which do not pose to be a difficult airway on preliminary clinical airway examination but often turn out to be so when handling the airway (the unanticipated difficult airway: the incidence being 9\% [6] - 13\% [7]).

The ASA difficult airway algorithm [8] recommend using a laryngeal mask airway to secure ventilation and oxygenation (after failed attempts of optimized direct laryngoscopy and change of laryngoscope blades) and also as an intubation conduit with or without fiberoptic guidance in such cases [9]. Although various alternative options of intubation (fibreoptic intubation, light wand guided intubation, retrograde intubation, blind nasal intubation) have been described in difficult intubation scenario, we considered LMA to be the most familiar and accessible option in the given emergency scenario. LMA would aid as an intubation conduit and also maintain ventilation. The other options described require great clinical skills and experience before they can be used in emergency scenarios. Moreover, they were not available in our emergency set up.

Intubation via a laryngeal mask has been described as a "low skill" technique. Even then, many problems can occur especially difficulty with fiberscopy [10]. Regardless of the difficulties, the choice or size of tracheal tube is restricted by their passage through the lumen of the laryngeal mask. Moreover, smaller diameter tubes may also be short of length for positioning the cuff safely below the cords and connection of the ventilating circuit. The intubating LMA is a standard option for intubation among supraglottic devices in patients with difficult air- way. However, its use may be limited by difficult insertion sometimes due to limited mouth opening \& its unavailability [11]. The non-availability of intubating LMA in our EOR was the limiting factor in this case.

The placement of appropriate sized ProSeal-LMA was not possible on two attempts in this case. Though the exact reason for unsuccessful placement is not clear, increased airway secretions and mucosal trauma from previous intubation attempts, inadequate relaxation (weaning of the effect of succinylcholine), relatively large epiglottis, and folding of Proseal LMA could be the contributing factors. Although the bag mask ventilation was adequate after failed intubation and Proseal LMA insertion events, the nature and duration of surgery necessitated airway device placement. An appropriately sized classic and intubating LMA have been successfully used in maintaining ventilation in such cases. These were however not available in the given scenario. I-gel when compared with other supraglottic devices for testing the ease of insertion into airway training manikins was found to be the best performing device [12]. This was also evident in this case from an easier insertion (in single attempt) than Proseal LMA and i-gel serving as a successful ventilation device.

The i-gel supraglottic airway has been used for ventilation and intubation in simulated difficult airway in manikin studies $[3,4]$. There are only three reports of its use in anticipated difficult airway. Michalek et al have used it for fibreoptic intubation in two patients with intellectual difficulty [2] while Sharma et al. have used it as an intubation device in a patient with previous history of difficult intubation [13]. A literature search, however, did not reveal any reported use of i-gel as an intubation conduit for unanticipated difficult airway and for emergency surgery, where attempts to intubation using different laryngoscope blades, repositioning and insertion of other supraglottic devices as intubation conduit failed and i-gel served as a rescue intubation device.

The main reason for inserting a supraglottic device as a conduit for tracheal intubation is provision of a patent airway and thus facilitating ventilation and oxygenation $[9,13]$. I-gel has been found to be a reasonable alternative to the LMA-Unique for controlled ventilation during anaesthesia [14]. It can be used during both spontaneous and intermittent positive pressure ventilation in anaesthetized patients [1]. Also, it has been associated with successful and easy insertions with short insertion times [14, 15] even with minimal training $[15,16]$. When tested for ease of insertion into airway training manikins, it has been found to be the best performance device in comparison to other supraglottic devices [12], which was also evident in this case.

Sharma and colleagues [13] have raised concerns over removal of i-gel after intubation as almost impossible. 
However, the diagnosis of perforation peritonitis necessitated the removal of i-gel after intubation to facilitate passage of a wide nasogastric tube, which would not have been possible through the limited sized lumen of the gastric drain channel of i-gel. The igel was removed using the "tube within the tube" assembly [5] and did not pose any difficulty to us.

The ASA difficult airway algorithm advocates the use of laryngeal mask airway in cases of unsuccessful face mask ventilation, as intubation conduit in non-emergent pathway and for conduct of surgery when multiple attempts to intubation have failed in the non emergent scenario. We found i-gel to be more successful and easier to insert than LMA and it being a useful intubation conduit when other available alternative intubation approaches were unsuccessful. It may thus be useful in averting "cannot ventilate cannot intubate" situation especially when LMA ventilation is inadequate. Our report along with the findings of previous authors [1-4,12,1416] suggests that i-gel could possibly be an alternative to LMA in the ASA difficult airway algorithm, though this requires further validation.

Thus, i-gel may possibly be another tool in the armamentarium of the emergency physicians for management of unanticipated difficult airway in emergency scenarios where other supraglottic devices are unavailable or have failed. Its usefulness in difficult airway situations may possibly find it a place in the difficult airway algorithm.

\section{REFERENCES}

[1] D. A. Gabbott and R. Beringer, "The iGEL Supraglottic Airway: A Potential Role for Resuscitation?” Resuscitation, Vol. 73, No. 1, pp. 161-164. doi:10.1016/j.resuscitation.2006.10.026

[2] P. Michalek, P. Hodgkinson and W. Donaldson, "Fiberoptic Intubation through an I-Gel Supraglottic Airway in Two Patients with Predicted Difficult Airway and Intellectual Disability,” Anesthesia \& Analgesia, Vol. 106, No. 5, 2008, pp. 1501-1504. doi:10.1213/ane.0b013e31816f22f6

[3] P. Michalek, W. Donaldson, C. Graham and J. D. Hinds, "Comparison of the I-Gel Supraglottic Airway as a Conduit for Tracheal Intubation with the Intubating Laryngeal Mask Airway: A Manikin Study,” Resuscitation, Vol. 81, No. 1, 2010, pp. 74-77. doi:10.1016/j.resuscitation.2009.10.009

[4] L. G. Theiler, M. Kleine-Brueggeney, D. Kaiser, N. Urwyler, C. Luyet, A. Vogt, R. Greif and M. Unibe, "Crossover Comparison of the Laryngeal Mask Supreme(TM) and the IGel(TM) in Simulated Difficult Airway Scenario in Anesthetized Patients,” Anesthesiology, Vol. 111, No. 1, 2009,

\section{pp. 55-62. doi:10.1097/ALN.0b013e3181a4c6b9}

[5] K. Chaudhary, L. Gupta and P. Bhadoria, "I-Gel Removal Made Easy: A 'Tube within the Tube’ Assembly,” E-Letter BJA, Oxford, 2011.

[6] G, Iohom, M. Ronayne and A. J. Cunningham, "Prediction of Difficult Tracheal Intubation,” European Journal of Anaesthesiology, Vol. 20, No. 1, 2003, pp. 31-36. doi:10.1097/00003643-200301000-00006

[7] J. C. Tse, E. B. Rimm and A. Hussain, "Predicting Difficult Endotracheal Intubation in Surgical Patients Scheduled for General Anaesthesia: A Prospective Blind Study," Anesthesia \& Analgesia, Vol. 81, No. 2, 1995, pp. 254-258.

[8] American Society of Anesthesiologists Task Force on Management of the Difficult Airway, "Practice Guidelines for Management of the Difficult Airway: An Updated Report by the American Society of Anesthesiologists Task Force on Management of the Difficulty Airway,” Anesthesiology, Vol. 98, No. 5, 2003, pp. 1269-1277.

[9] H. S. Joo and K. D. Rose, "The Intubating Laryngeal Mask Airway with and without Fiberoptic Guidance," Anesthesia \& Analgesia, Vol. 88, No. 3, 1999, pp. 662-666.

[10] A. Higgs, E. Clark and K. Premroj, "Low Skill Fiberoptic Intubation: Use of the Aintree Catheter with the Classic LMA,” Anaesthesia, Vol. 60, No. 9, 2005, pp. 915-920. doi:10.1111/j.1365-2044.2005.04226.x

[11] G. Caponor, "Intubating laryngeal Mask Airway,” Anaesthesia and Intensive Care, Vol. 30, 2002, pp. 551-569.

[12] K. M. Jackson and T. M. Cook, "Evaluation of Four Airway Training Manikins as Patient Simulators for the Insertion of Eight Types of Supraglottic Airway Devices,” Anaesthesia, Vol. 62, No. 4, 2007, pp. 388-393. doi:10.1111/j.1365-2044.2007.04983.x

[13] S. Sharma, M. Popat and R. Rogers, "The I-Gel ${ }^{\mathrm{TM}}$ Airway for Ventilation and Rescue Intubation,” Anaesthesia, Vol. 62, No. 4, 2007, pp. 419-420. doi:10.1111/j.1365-2044.2007.05045.x

[14] V. Uppal, S. Gangaiah, G. Fletcher and J. Kinsella, "Randomized Crossover Comparison between the I-Gel and the LMA-Unique in Anaesthetized, Paralysed Adults," British Journal of Anaesthesia, Vol. 103, No. 6, 2009, pp. 882-885. doi:10.1093/bja/aep292

[15] N. M. Wharton, B. Gibbison, D. A. Gabbott, G. M. Haslam, N. Muchatuta and T. M. Cook, "I-Gel Insertion by Novices in Manikins and Patients,” Anaesthesia, Vol. 63, No. 3, 2008, pp. 991-995. doi:10.1111/j.1365-2044.2008.05542.x

[16] L. Beylacq, M. Bordes, F. Semjen and A.-M. Cros, “The I-Gel, a Single-Use Supraglottic Airway Device with a Non-Inflatable Cuff and an Esophageal Vent: An Observational Study in Children," Acta Anaesthesiologica Scandinavica, Vol. 53, No. 3, 2009, pp. 376-379. doi:10.1111/j.1399-6576.2008.01869.x 